ro list an event in the Calendar, :ontact J. Dininny, Materials Pesearch Society, 9800 McKnight Road, Suite 327, Pittsburgh, PA 15237; (412) 367-3036; fax (412) 367-4373.

M|R|S indicates the meeting is sponsored by or affiliated with the Materials Research Society.

See MRS BULLETIN Vol. XIII No. 11 for Calendar events from December 1 through December 31, 1988.

\section{JANUARY 1989}

2-7

Second International Symposium on Solid State Physics Kandy, Sri Lanka

Institute of Fundamental Studies Hantana Rd., Kandy, Sri Lanka: (08) 32002; fax 0832001

4-6

Short Course on X-Ray Diffractometry \& Topography of Semiconducting Materials \& Epitaxial Thin Films

San Diego, CA

North Eastern Analytical Corp. 17

Sherman Ro. P.O. Box 25, Millis, MA 02054; (508) 376-4132

9-12

Composites in Manufacturing 8 Anaheim, CA

K.A. Wilson, Society of

Manufacturing Engineers. One

SME Dr., P.O. Box 930, Dearborn

MI 48121: (313) 271-0777

$\operatorname{fax}(313) 271-2861$

11-13

SC Global '89

San Francisco. CA

Superconductor Applications

Association, 24781 Camino Villa

Ave., El Toro, CA 92630; (800)

854-8263

15-18

13th Annual Conference on

Composites and Advanced

Ceramics

Cocoa Beach, FL

American Ceramic Society, 757

Brooksedge Plaza Dr., Westerville

$\mathrm{OH}$ 43081-6136; (614) 890-4700;

$\operatorname{tax}(614) 899-6109$

$15-20$

O-E/LASE '89: Optoelectronics

and Laser Applications in Science

and Engineering

Los Angeles, CA

SPIE, P.O. Box 10, Bellingham.

WA 98227-0010: (206) 676-3290

23-24

Second Annual Cambridge

Conference on Commercial

Applications of Superconductivity

Boston, MA

World Tech Press, One Kendall

Square, Suite 2200, Cambridge,

MA 02139; (617) 661-0510
$30-2$

CeramTec ' 89

T.J. Whalen, Engineering Society of Detroit, Regional Conference

Center, 24 Frank Lloyd Wright Dr.,

Suite C-500, Ann Arbor, MI 48105.

(313) 995-4440; $\operatorname{tax}(313$ ) 663-7835

30-4

Winter European Course on

Amorphous Silicon

Folgaria, Italy

G. Thiels, Winter European

Courses, TASK R\&S S.r.I., Via

Verbano 2, 21027 Ispra (VA), Italy;

(0332) $781768 / 71 / 77$

$\operatorname{tax}(0332) 780802$

31-3

17th Australian Polyme

Symposium, Polymers in a Hostile Environment

Brisbane, Australia

D.J.T. Hill, Chemistry Dept.

University of Queensland

Brisbane, 4067, Australia

31-3

International Conference on

Sintering of Multiphase Metal and

Ceramic Systems

New Delhi, India

G.S. Upadhyaya, Convener

ICSMC '89, Dept. of Metallurgical

Engineering. Indian Institute of

Technology, Kanpur-208 016.

India: 240066 , ext. 933

\section{FEBRUARY 1989}

6-8

Conference on Integrated and

Guided-Wave Optics (IGWO)

Topical Meeting

Houston, TX

Optical Society of America, 1816

Jefferson PI. NW, Washington, DC

20036; (202) 223-0920

6-9

Conference on Optical Fiber

Communication (OFC '89)

Houston, TX

Optical Society of America, 1816

Jefferson PI. NW, Washington, DC

20036: (202) 223-0920

7-9

PCSI-16: Physics and Chemistry

of Semiconductor Interfaces

Bozeman, MT

G.J. Lapeyre, Physics Dept.,

Montana State University.

Bozeman MT 59717

(406) 994-6153

7-9

Superconductive Electronics

Short Course

UCLA, Los Angeles, CA

UCLA Extension, P.O. Box 24901

Los Angeles, CA 90024;

(213) 825-1047
13-17

Fifth International Wire Chamber

Conference

Vienna, Austria

M. Regler, Institut für

Hochenergiephysik der OeAW

Nikosdorfergasse 18, A-1050.

Vienna, Austria

21-24

Fourth International Symposium

on Recent Advances in Drug

Delivery

Salt Lake City, UT

Kristine Knutson, University of

Utah, Dept. of Pharma-

ceutics/CCCD. 421 Wakara Way,

Suite 318, Salt Lake City, UT

84108: (801) 581-6654

27-1

Microphysics of Surfaces, Beams

and Adsorbates Topical Meeting

Salt Lake City, UT

Optical Society of America, 1816

efferson PI. NW, Washington, DC 20036; (202) 223-0920

$27-2$

TMS Annual Meeting

Las Vegas, NV

The Metallurgical Society, 420

Commonwealth Dr. Warrendale.

PA 15086; (412) 776-9050

27-3

Gordon Conference on

Superconductivity

West Coast, USA

A. Sleight, Experimental Station $356 / 301$, E.I. du Pont de Nemours \& Co., Wilmington, DE 19898 (302) 695-3536

\section{MARCH 1989}

$6-8$

Fourth International Seminar on

Lithium Battery Technology and

Applications

Deerfield Beach, FL

S.P. Wolsky, Ansum Enterprises.

950 De Soto Rd., Suite 3B, Boca

Raton, FL 33432; (407) 391-3544

6-9

\begin{tabular}{|l|l|l|}
\hline $\mathbf{R}$ & $\mathbf{S}$ \\
\hline
\end{tabular}

Advanced Materials Conference

Denver, CO

F. Smith. Advanced Materials

Institute, Colorado School of

Mines, Golden, CO 80401 ;

(303) 491-8657

(See related article in Vol. XIIINo. 5 .)

6-9

Ninth General Conference,

Condensed Matter Division. EPS

Nice, France

J.P. LaHeuorte, Lab. de la Metiere

Condensee, Univ, de Nice.

F-06034 Nice, Cedex, France

8-9

Molecular Chemistry for Solid-

State Electronics

London, United Kingdom

The Royal Society, 6 Cariton

House Terrace, London SW1Y

5AG, United Kingdom
12-25

NATO Advanced Study Institute:

Diffusion in Materials

Aussois, France

A.L. Laskar, Dept. of Physics and

Astronomy. Clemson Univ.

Clemson, SC 29631:

(803) 656-5310; $\operatorname{tax}(803) 656-4040$

20-22

European Workshop on

Refractory Metals and Silicides

Houthalen, Belgium

R. De Keersmaecker, IMBE

Kapeldreef $75,8-3030$ Leuven,

Belgium; (32) 16281211

(See related article in Vol. XIII NO. 7.)

20-24

American Physical Society

Meeting

St. Louis, MO

W.W. Havens. American Physical

Society, 335 E. 45th St., New York,

NY 10017: (212) 682-7341

27-31

1989 Technical Symposia on

Aerospace Sensing including

Symposium on Infrared Applied

Science \& Engineering and

Symposium on Optics, Electro-

Optics, \& Sensors

Orlando, FL

SPIE, P.O. Box 10, Bellingham

WA 98227-0010: (206) 676-3290

$\operatorname{fax}(206) 647-1445$

29-31

Neutron and X-Ray Scattering

Complementary Techniques

Canterbury, United Kingdom

R.J. Newport. Physics Lab.,

University of Kent, Canterbury

CT2 7NR, United Kingdom

30-31

Colorado Microelectronics

Conference

Colorado Springs, CO

CMC ' 89 Conference Secretary.

Microelectronics Research

Laboratories, Univ. of Colorado.

Colorado Springs, $\mathrm{CO}$

80933-7150; (719) 593-3488

30-10

NATO Advanced Study Institute:

International Advanced Course on

Frontiers of Laser Spectroscopy

of Gases

Vimelro, Portugal

J.M. Hollas, Dept. of Chemistry,

University of Reading, Reading

RG6 2AD, United Kingdom

30-14

Experimental Workshop on High

$T_{c}$ Superconductors

Trieste, Italy

International Centre for

Theoretical Physics, HP.O. Box

586, 1-34100 Trieste, Italy: (0-40)

224241; fax 224531 


\section{APRIL 1989}

1-6

World Congress on

Superconductivity

Houston, TX

R.D. Kane, P.O. Box 27805

Houston, TX 77227-7805;

(713) $895-2500$

3-6

M RS Fifth Conference on Low

Energy Ion Beams

Guildford, United Kingdom

Meetings Office. The Institute of

Physics, 47 Belgrave Sq., London

SWIX BOX, United Kingdom

01-235-6111

4-7

Fifth National Space Symposium Colorado Springs, $\mathrm{CO}$

U.S. Space Foundation, P.O. Box

1838, Colorado Springs, $C O$

80901; (719) 550-1000

4-7

British Crystallographic

Association Spring Meeting

Oxford, United Kingdom

M. Glazer, Dept. of Physics

Clarendon Laboratory, Parks

Road, Oxford, OX1 3PU, United

Kingdom; 0865-272290

4-7

Electrochemical Processing of

Semiconductors

Berlin. W. Germany

W. Pieth, Free University of Berlin

Institute of Physical Chemistry

Takustrasse 3, D-1000 Berlin 33

6-7

NATO Workshop on Modeling in

Crystal Growth from the Melt

Parma, Italy

G. Müller, Institut für

Werkstoffwissenschaften 6

Martensstrasse 7, D-8520

Erlangen, W. Germany

(See related article in Vol. XIII No. 7. )

9-14

American Chemical Society

Meeting

Dallas, TX

American Chemical Society, 1155

16th St. NW. Washington, DC

20036; (202) 872-4600

10-13

MRIS Sixth Oxford Conterence on Microscopy of Semiconducting Materials

Oxford, United Kingdom

Administrator, Royal

Microscopical Society, 37/38 St.

Clements, Oxford OX4 1AJ

United Kingdom:

44(0)-865-248768

(See relatedarticle in Vol. XIII No. 9.)

17-21

International Conference on

Metallurgical Coatings

San Diego, CA

B.D. Sartwell, Naval Research

Lab, Code 4675, Washington, DC

20375; (202) 767-4800;

$\operatorname{fax}(202) 767-5301$
23-27

91st Annual Meeting and Expo of the American Ceramic Society

indianapolis, IN

American Ceramic Society, 757

Brooksedge Plaza Dr., Westerville

$\mathrm{OH} 43081-6136$; (614) 890-4700:

$\operatorname{fax}(614) 899-6109$

\section{3-28}

Nondestructive Testing

Amsterdam, Netherlands

12th WCNDT ' 89 , B. de Jong, RAI

Organisatie Bureau Amsterdam

bv, Europaplein 12, $1078 \mathrm{GZ}$

Amsterdam, Netherlands:

(31) 20-549-12-12;

$\operatorname{tax}(31)$ 20-46-44-69

\section{$24-28$}

\section{M|RTS Materials Research}

Soclety Spring Meeting

Town and Country Hotel, San

Diego, CA

M. Geil, Materials Research

Society, 9800 McKnight Rd., Suite

327, Pittsburgh, PA 15237;

(412) 367-3003; fax (412) 367-4373

24-28

Conference on Lasers and

Electro-Optics (CLEO '89)

Baltimore, MD

Optical Society of America, 1816

Jefferson PI. NW, Washington, DC

20036; (202) 223-0920

24-28

Second International Congress on

Optical Science and Engineering

Paris, France

SPIE, P.O. Box 10, Bellingham,

WA; (206) 676-3290

24-28

Quantum Electronics and Laser

Science Conference (QELS '89)

Baltimore, MD

Optical Society of America, 1816

Jefferson PI. NW, Washington, DC

20036; (202) 223-0920

\section{MAY 1989}

1-4

American Physical Society Spring

Meeting

Baltimore, MD

American Physical Society, 335

East 45th Street, New York, NY

10017; (212) 682-7341

7-12

Symposium on High $\mathrm{T}_{\mathrm{C}}$

Superconductor Technologies

(HiT ST2) at the 175th Meeting of

The Electrochemical Society

Los Angeles, CA

S. Raider, IBM T.J. Watson

Research Center, P.O. Box 218

Yorktown Heights, NY 10598;

(914) 945-2203

7-12

Third International Conference on

Fundamentals of Adsorption

Nurrigen, W. Germany

DECHEMA, Abt. Tagungen, P.O

Box 970146, TheodorHeuss-Allee

25. D-6000 Frankfurt am Main 97,

W. Germany 11th Oak Ridge Nationa

8-11

34th SAMPE International

Symposium and Exhibition

Reno, NV

SAMPE, 843 West Glentana, P.O.

Box 2459, Covina, CA 91722; (818)

$331-0616$

8-12

aboratory Symposium on

Biotechnology for Fuels and

Chemicals

Colorado Springs, CO

E. Greenbaum, Chemical

Technology Div., Oak Ridge

National Lab., 4500 N, MS 6194 .

P.O. Box 2008 , Oak Ridge, TN

37831-6194; (615) 574-6835

9-11

International Conference on

Coatings and Sensors for

Acoustic and Electromagnetic-

Optical Applications

University Park, PA

V.K. Varadan, Pennsylvania State

University, Dept. of Engineering

Science and Mechanics,

University Park, PA 16802;

(814) $865-2410$

$16-19$

Swift Heavy lons in Matter

Caen, France

Symposium Secretary, SHIM

89/CIRIL, BP 5133, 14040 Caen

Cedex, France; 31454639

fax 31454665

22-25

Fifth International Symposium on

Graphite Intercalation

Compounds

Berlin, W. Germany

F.W. Froben, Fachbereich Physik

Freie Universität Berlin, Arnimalle

14, D-1000 Berlin 33, W. Germany; (030) $8384235 / 5566$

22-26

Acoustical Society of America

117th Meeting

Syracuse, NY

Acoustical Society of America,

335 East 45th St. New York, NY

$10017 ;$ (212) $661-9404$

30-2

E-M|R|S 1989 E-MRS Spring

Meeting

Strasbourg, France

P. Siffert, Centre de Recherches

Nucléaire, Laboratoire PHASE,

F-67037, Strasbourg Cedex

France: 88286543

30-2

$E-M|R| S$ Course on High Tech

Blomaterlals

Strasbourg, France

D. Muster, L.E.E.D. Biomateriaux

CHRU-BP 426, F-67091

Strasbourg Cedex, France

(See relatedarticlein Vol. XIII, No. 11.)

31-2

11th Symposium on Applied

Surface Analysis

Cleveland, $\mathrm{OH}$

J. Grant, Research Institute, 300

College Park, Dayton, OH 45469 0001; (513) 255-5125 or G.

Chottiner, Dept of Physics, Case

Western Reserve, Cleveland, $\mathrm{OH}$

44106; (216) 368-4024

\section{JUNE 1989}

15-29

Advances in Nonradiative

Processes-NATO Advanced Study Institute

Erice, Italy

B. DiBartolo, Dept, of Physics,

Boston College, Chestnut Hill, MA 02167; (617) 552-3575

20-22

SAMPE-Third International

Electronics Conference

Los Angeles, CA

M. Smith, Business Director.

SAMPE, P.O. Box 2459, Covina

CA 91722: (818) 331-0616

21-23

Electronic Materials Conference

Cambridge, MA

The Metallurgical Society, 420

Commonwealth Dr., Warrendale,

PA 15086: (412) 776-9050

26-2B

Coherence and Quantum Optics

Conference

Rochester, NY

L. Mandel, University of

Rochester, Dept. of Physics.

Rochester, NY 14627;

(716) 275-4361

\section{JULY 1989}

10-14

International Conference on Ion

Sources

Berkeley, CA

W. O'Connor, Lawrence Berkeley

Lab., 50B-2270, Berkeley, CA

94720;

(415) 486-6386; $\operatorname{fax}(415)$ 486-7000

10-14

Fourth International Conference

on Electron Spectroscopy

Honolulu, $\mathrm{HI}$

G.E. McGuire, Microelectronics

Center of North Carolina,

P.O. Box 12889, Research

Triangle Park, NC 27709;

(919) 248-1800; or C.R. Brundle.

IBM Almaden Research Center.

650 Harry Rd., San Jose, CA

95120; (408) 927-2444

12-14

Solid State Lasers Topical

Meeting/Sino-Japanese Joint

Meeting on Optical Fiber Science

and Electromagnetic Theory ' 89

Beijing. China

Optical Society of America, 1816

Jefferson PI. NW, Washington, DC

20036; (202) 223-0920 
17-21

Ninth International Conference on Vacuum Ultraviolet Radiation Physics

Honolulu, HI

C.S. Fadley, Dept. of Chemistry, Univ. of Hawaii, 2545 The Mall.

Honolulu, HI 96822;

(808) $948-6401$

18-21

Seventh International Conference on Integrated Optics and Optical Fiber Communication

Kobe, Japan

Secretariat, $100 C^{\prime} 89, \mathrm{c} / 0$

Business Center for Academic

Societies Japan, Conference

Dept., Yamazaki Bldg. 4F, 2-40-14,

Hongo, Bunkyo-ku, Tokyo 113.

Japan

23-24

International Symposium on

Computer-Aided Materials

Characterization

Charlotte, NC

R. Biederman, Worcester

Polytechnic Institute, Worcester

MA 01609; (508) 831-5453/5346

23-28

American Crystallographic

Association Annual Meeting

Seattle, WA

American Crystallographic

Association, 335 E. 45th St., New

York, NY 10017; (212) 661-9404

23-28

International Conference on

Materials and Mechanisms of

Superconductivity: High

Temperature Superconductors

(HTSC-M²S)

Stanford, CA

$J$. Higgins, Meeting Planning

Associates, 883 Santa Cruz Ave.

Menlo Park, CA 94025:

(415) 326-7781; fax (415) 326-3945

24

Short Course on Quality and

Reproducibility of Image Analysis

Data

Charlotte, NC

I. LeMay, Metallurgical Consulting

Services, Ltd. P.O. Box 5006

Saskatoon, Saskatchewan,

Canada S7K 4E3; (306) 244-4535

24-26

Second Micro-Optics

Conference/Eighth Topical

Meeting on Gradient-Index

Optical Imaging Systems

Tokyo, Japan

K. Iga, Tokyo Institute of

Technology, Research Laboratory

of Precision Machinery and

Electronics, 4259 Nagasuta,

Midori-ku, Yokohama 227, Japan

25-26

International Conference on

Computer-Aided Microscopy and

Quality Control

Charlotte, NC

J. Braun, EG\&G Mound Applied

Technologies, Miamisburg, OH

45343-0987; (513) 865-3829
24-28

Cryogenic Engineering

Conference and International

Cryogenic Materials Conference

Los Angeles, CA

D. Hustvedt, CEC/ICMC

Secretariat, Dept. of Chemical

Engineering $\mathrm{BH}$ 5405, University

of California, Los Angeles, CA 90024-1592

31-3

Sixth International Meeting on

Photoacoustic and Photothermal

Phenomena

Baltimore, MD

J. Murphy, Johns Hopkins Applied

Physics Lab, Johns Hopkins Rd.

Laurel, MD 20707; (301) 953-6214

31-4

36th International Field Emission

Symposium

Oxford, United Kingdom

Administrator, Royal

Microscopical Society, 37/38 St.

Clements, Oxford, OX4 1AJ, U.K.; 865-248768

(See related article Vol. XIII No. 11.)

\section{AUGUST 1989}

2-7

32nd International Union of Pure and Applied Chemistry Congress Stockholm, Sweden

IUPAC, c/o Stockholm

Convention Bureau, P.O. Box

6911, S-10239 Stockholm,

Sweden; 468230990

fax 468348441

13-26

NATO Advanced Study Institute:

High $T_{C}$ Superconductors-

Physics and Materials Science

Bad Windsheim, W. Germany

R. Kossowsky, Applied Research

Lab., Pennsylvania State Univ..

P.O. Box 30, State College, PA

16804; (814) 863-4481

28-1

Third International Conference on

Surface Modification

Technologies

Neuchatel, Switzerland

The Metallurgical Society, 420

Commonwealth Dr., Warrendale,

PA 15086; (412) 776-9050

28-1

12th International Congress on

$\mathrm{X}$-Ray Optics and Microanalysis Kracow, Poland

S. Jasienska. Academy of Mining

and Metallurgy, Institute of

Metallurgy, 30 059, Kracow.

Poland

29-2

International Symposium on Innovation and Perspectives in

Solid Phase Synthesis

Oxford, England

R. Epton, School of Applied

Sciences, Wolverhampton

Polytechnic, Wolverhampton WV1

1SB, England

\section{SEPTEMBER 1989}

4-6

Fourth MRM Conference

Rimini, Italy

L. Pareti, Instituto Maspec CNR

Via Chiavari 18/A 43100 Parma

Italy; 521-95811; fax 521-96315

4-8

Macromolecules '89: Second

Euro-American Conference on

Functional Polymers and

Biopolymers

Oxford, England

R. Epton, School of Applied

Sciences, Wolverhampton

Polytechnic, Wolverhampton WV 1 TSB, England

5-7

International Conference on Applications of Supercomputers

in Engineering

Southampton, United Kingdom

L. Newman, Computational

Mechanics Institute, 52 Henstead

Rd., Southampton, S01 2DD,

England; 44-703-221398;

fax 44-703-331020

$5-7$

International Conference on

Interfacial Phenomena in

Composite Materials

Sheffield, United Kingdom

J. Herriot, Conference Organizer

Butterworth Scientific Ltd., P.O.

Box 63, Westbury House, Bury St.,

Guildford, Surrey GU2 5BH.

United Kingdom; 0483-300966

5-9

First European Conference on

Accelerators in Applied Research

Technology (ECCART)

Frankfurt am Main, W. Germany

$K$. Bethge, Institut für Kernphysik,

August-Euler-Str 6, D-6000

Frankfurt am Main 90;

069-7 $984242 / 44$

6-9

European Magnetic Materials and Applications ' 89 Conference

Rimini, Italy

L. Pareti, Local Chairman EMMA

'89, Istituto Maspec CNR, Via

Chiavari 18/A, 43100 Parma, Italy;

521-95811; fax 521-96315

6-15

International School of Plasma

Physics-Tritium and Advanced

Fuels in Fusion Reactors

Varenna, Italy

E. Sindoni, International School of

Plasma Physics, 16 Via Celoria,

20133 Milano, Italy; 2392267 ;

26650 05; fax 223-66583

10-15

198th American Chemical Society

National Meeting

Miami Beach, FL

Meetings Dept., American

Chemical Society, 1155 16th St

NW, Washington, DC 20036:

(202) 872-4396

11-15

European Conference on Optical

Communication

Gothenburg, Sweden

ECOC ' 89 Secretariat, Chalmers

University of Technology, Dept. of

Electrical Measurements,

Research Laboratory of Electro-

Optics and Laser, S-41296

Gothenburg. Sweden

$17-20$

12th Biennial ASME Conference

on Mechanical Vibration and

Noise

Montreal, Canada

T.S. Sankar, Dept. of Mechanical Engineering, S-B 302, Concordia

University, 1455 de Maisonneuve

Blvd., Montreal, Quebec, Canada

H3G 1M8; (514) 848-3130/3160

$17-22$

Conference on Productivity and

Technology in the Metallurgical

industries

Cologne, W. Germany

B. Kamperman, The Metallurgica

Society, 420 Commonwealth Dr.

Warrendale, PA 15086:

(412) 776-9050

17-22

TMS Fall Meeting: Extractive and

Process Metallurgy

Cologne, W. Germany

The Metallurgical Society, 420

Commonwealth Dr., Warrendale,

PA 15086; (412) 776-9050

18-22

American Society for Precision

Engineering Annual Meeting and

Fifth International Precision

Engineering Seminar

Monterey, CA

G. Swingle, ASPE/IPES5

Conference Office, P.O. Box 808

L-292, Livermore, CA 94550; (415)

422-5447; fax (415) 423-2419

20-22

Second International Conference

on Structural Adhesives in

Engineering II

Bristol, United Kingdom

J. Herriot, Conference Organizer,

Butterworth Scientific Ltd., P.O.

Box 63, Westbury House, Bury St.

Guildford, Surrey GU2 5BH,

United Kingdom; 0483-300966

25-29

11 th International Vacuum

Congress (IVC-11) and 7th

International Conference on Solid

Surfaces (CSS-7)

Cologne, W. Germany

D.A. Benninghoven,

Physikalisches Institut Der

Universtat Munster Wilhelm-

Klemm Strasse 10, D-4000

Munster, W. Germany

26-28

21st international SAMPE

Technical Conference

Atlantic City, NJ

SAMPE, 843 W. Glentana, P.O.

Box 2459, Covina, CA 91722; (818) 


OCTOBER 1989
1-5
TMS Fall Meeting: Physical
Metallurgy and Materials
Indianapolis, IN
The Metallurgical Society, 420
Commonwealth Dr., Warrendale,
PA 15086; (412) 776-9050
2-4
Second European Conference on
Electron and Optical Beam
Testing of Integrated Circuits
Duisburg, W. Germany
E. Kubalek and L.J. Balk,
Werkstoffe der Elektrotechnik,
Universitat Duisburg,
Kommandantenstr. 60 D-4100
Duisburg 1. W. Germany;
49-203 379 34 06
2-6
European Space Power
Conference
Madrid, Spain
J-C. Larue, European Space
Research and Technology Centre,
XPG. Postbus 299,2200 AG
Noordwijk ZH, Netherlands: 31 (0)
1719 83973; fax 171917400

15-20

OSA Annual Meeting

Orlando. FL

Optical Society of America, 1816

Jefferson PI. NW, Washington, DC

20036; (202) 223-0920

21-24

Soil Dynamics \& Earthquake

Engineering IV Conference

Mexico City, Mexico

L. Newman, Computational

Mechanics Institute, 52 Henstead

Rd., Southampton, S01 2DD,

England; 44-703-221398;

fax 44-703-331020

22-28

Second International Conference

on Solid State \& IntegratedCircuit

Technology

Beijing, China

Continuing Education in

Engineering. University Extension,

University of California, 2223

Fulton St., Berkeley, CA 94720 ;

(415) 642-4151; fax (415) 643-8683

(Seerelatedarticle in Vol. XIIINo. 11.)
23-27

36th National Vacuum Symposium of the AVW

Boston, MA

M. Churchill, American Vacuum

Society, 335 E. 45th St., New York,

NY 10017 ; (212) 661-9404

24-29

European Conference on

Applications of Surface and

Interface Analysis (ECASIA 89)

Antibes, France

$J$. Fauvet, Sociéte Français du

Vide, 19 rue du Renard, F-75004

Paris, France

\section{NOVEMBER 1989}

27-2

M] R]S Materials Research

Sociely Fall Meeting

Boston Marriott and Westin

Hotels/Copley Place, Boston, MA

M. Geil, Materials Research

Society, 9800 McKnight Rd., Suite

327, Pittsburgh, PA 15237;

(412) $367-3003 ;$ fax (412) $367-4373$

\section{DECEMBER 1989}

14-26

Royal Australian Chemical

Institute PAC-Chem 89

International Congress of Pacific

Basin Chemical Societies

Hawaii

D.J.T. Hill, Chemistry Dept.,

University of Queensiand, St.

Lucia, Queensland, 4067.

Australia

\section{State-of-the-art techniques will be detailed in these 1 -day courses sponsored by the Structural Materials Division.}

\section{FUNDAMENTALS OF FAILURE ANALYSIS}

\section{Saturday, February 25}

Instructor: Dr. Andrew Madeyski, Westinghouse R\&D

Step-by-step instruction for conducting failure analysis will be presented, focussing on methods, techniques and instruments, as well as possible pitfalls to avoid.

\section{PRINCIPLES OF SURFACE MODIFICATION ENGINEERING \\ Saturday, February 25 \\ Instructor: Prof. J. Michael Rigsbee, University of Illinois}

Principles and applications of surface engineering, including conventional techniques such as carburizing, and evolving processes such as physical vapor deposition and laser hardening.

\section{MATERIALS CHARACTERIZATION TECHNIQUES: IONS, PHOTONS \& ELECTRONS \\ Saturday, February 25 \\ Instructor: Prof. Ram Kossowsky, Penn State University}

Three major classes of analytical techniques and instruments based on photon, electron or ion radiations will be presented, with emphasis on limitations, advantages, sensitivities, resolutions, and applications to the analyses of metals, ceramics and polymers.
DESIGN PRINCIPLES OF COMPOSITE MATERIALS

\section{Sunday, February 26}

Instructor: Prof. Maurice Amateau, Penn State University

Basic definitions, design principles and constituent properties of metal, ceramic and polymer composites, as well as special topics such as joining, mechanical performance, thermal effects and applications.

\section{APPLICATIONS OF ARTIFICAL INTELLIGENCE TO ENGINEERING \\ Sunday, February 26 \\ Instructor: Dr. Jan Schreurs, Westinghouse R\&D}

Measures of intelligence, search strategies, heuristics, and knowledge representations will be discussed, as well as applications to problems in industry.

\section{FUNDAMENTALS OF STRUCTURE \& MECHANICAL PROPERTIES OF POLYMERS: \\ A MATERIALS SCIENCE APPROACH \\ Sunday, February 26}

Instructor: Prof. Stephen H. Carr, Northwestern University

The effect of microstructure on mechanical behavior of polymeric materials will be discussed, with emphasis on semicrystalline polymers, block copolymers, and polymer alloys.

All Courses: $\$ 295$ each

For information, contact: Continuing Education. TMS. 420 Commonwealth Drive. Warrendale. PA 15086 - Telephone (412)776-9011 